\title{
Visualization of enrollment data using chord diagrams
}

\author{
Laia Blasco-Soplón ${ }^{1}$, Josep Grau-Valldosera ${ }^{2}$ and Julià Minguillón ${ }^{1}$ \\ ${ }^{1}$ Computer Science, Multimedia and Telecommunication Studies, Universitat Oberta de Catalunya, Rambla Poblenou 156, \\ Barcelona, Spain \\ ${ }^{2}$ Marketing Department, Universitat Oberta de Catalunya, Rambla Poblenou 156, Barcelona, Spain \\ \{lblascos, jgrauv, jminguillona $\} @$ uoc.edu
}

Keywords: Enrollment, Dropping out, Chord diagrams, Radial visualization, Distance education, Higher education

Abstract: Distance and online universities have usually more flexible academic requirements than brick-and-mortar ones, so students do not uniformly advance within the programme they enroll into as they are supposed to. Furthermore, due to their intrinsic nature, distance students need additional support for deciding which subjects they will take every semester. On the other hand, teachers have usually no idea about what other subjects are taking the students enrolled in their subjects. This paper proposes the use of chord diagrams for visualizing the relationships between pairs of subjects taken by students, with the aim of providing both university managers and students with a clear picture of possible bottlenecks, that is, combinations of subjects that might affect students' performance. Chord diagrams can be used to analyze intra-semester data (subjects taken simultaneously) and inter-semester data (sequences of subjects taken by students) and provide a fast overview of what is happening within a given programme at subject level. Furthermore, it is possible to interact with chord diagrams in order to filter and obtain additional subject details if desired.

\section{INTRODUCTION}

Enrollment and dropping out in higher education are two sides of the same coin. In the case of distance and online universities, dropping out mostly occurs after the end of the first academic semester or year (Grau-Valldosera and Minguillón, 2014; Tyler-Smith, 2006). In (Campbell et al., 2007), the authors describe several examples of learning analytics related to enrollment, some of them oriented towards improving student retention. Usually, all these studies are used-centered, trying to determine whether a student will be accepted or not, or trying to predict her academic performance. Although such data-driven decision support systems can be built using historical enrollment data (see (Sacín et al., 2011; Siraj and Abdoulha, 2009), for instance), we are more interested in obtaining a fast overview of what is happening within a given programme, adopting a subjectcentered approach and following the basic premises of information visualization (Card et al., 1999). In (Grau-Valldosera and Minguillón, 2014), the authors proposed a novel definition of dropping out adapted to the specific characteristics of distance universities, based on the minimum number of break semesters $(N)$ a student takes, needed to determine that such student never enrolls again (i.e. drops out), for a given error rate (less than $5 \%$ of students come back after $N$ semesters). Using such definition, the authors were able to compute the dropping out rate for different programmes, which lead to strong evidences: half of the dropouts occur after the first academic semester, and up to $75 \%$ of accumulated dropouts occur after the second semester (i.e. the first year). As most students take only two or three subjects during their first semester, they should know which combinations are known to be problematic, using accumulated enrollment data. Therefore, it is very important to provide both university managers and students with visualization tools that allow them to detect possible bottlenecks for a given programme and help them to adjust their expectations about subject enrollment, respectively. As stated in (Park and Choi, 2009), "internal factors such as subject design strategies and learners' motivation should be prioritized at the subject development stage in order to make the subject participatory and interesting and to keep learners engaged". We think that these strategies should be moved one level upward, trying to detect problems not only at subject level but at programme level, visualizing how students advance within programmes and the possible barriers caused by wrong enrollment decisions. 
This paper is structured as follows: the dataset with enrollment data used in this paper is analyzed in Section 2. Section 3 proposes the use of chord diagrams for visualizing subject relationships with respect to enrollment. Finally, conclusions and future work are stated in Section 4.

\section{ENROLLMENT DATA}

The dataset used in this paper is taken from Universitat Oberta de Catalunya (UOC) academic databases. Only valid enrollments have been included, i.e. ones that have been formalized and paid for, thus excluding enrollments that were subsequently canceled. As we are just exploring the possibility of using chord diagrams for visualizing relationships between subjects, we have chosen the largest programme amongst all available data, i.e., students enrolled into Economics between Spring 1999 and Spring 2011, containing data about 21792 students and 501 different subjects, giving a total of 328467 subject enrollments during 25 consecutive semesters.

Table 1 shows the expected sequence of subjects for the Economics programme. It is supposed to be finished in 6 semesters ( 3 years), taking 6 subjects every semester. Here x.x means subjects chosen from a pool of optional subjects or from other programmes. This structure is only a recommendation, so students can take subjects in any order and number. Actually, the only requirement is subject 00.010 (a basic subject on online competencies) which is mandatory for all students during the first semester. A more complete version of Table 1 is what students have before they decide which subjects they will enroll into, including information about each subject. As expected, due to the nature of distance students (most of them have a full-time work, family responsibilities, and so), they usually do not enroll into a complete semester (i.e. 6 subjects), but fewer. Furthermore, they do not even follow the predetermined order of subjects, that is, they can enroll into subjects from the second semester without having taken subjects from the first one. Therefore, the concept of cohort is completely undermined.

Table 2 partitiones students according to the number of subjects $(C)$ they enroll into, as well as the number of subjects they successfully pass. Notice that students take more subjects in average during the first semester than during the second one. This could be a sign that students learn to regulate their learning process by narrowing their enrollment once they have acquired the experience of being online learners. As stated in (Kiernan et al., 2004), the process of be-
Table 1: subjects and degree plans for the Economics programme.

\begin{tabular}{|c|c|c|c|c|c|c|}
\hline$S$ & \multicolumn{7}{|c|}{ subjects } \\
\hline 1 & 01.001 & 01.079 & 01.003 & 01.004 & 00.010 & 00.002 \\
\hline 2 & 01.005 & 01.006 & 01.007 & 01.078 & 01.009 & 00.003 \\
\hline 3 & 01.080 & 01.021 & 01.086 & 01.020 & 00.004 & x.x \\
\hline 4 & 01.014 & 01.015 & 01.012 & 01.087 & 01.011 & x.x \\
\hline 5 & 01.018 & 01.019 & 01.016 & 01.022 & x.x & x.x \\
\hline 6 & 01.008 & 01.023 & x.x & x.x & x.x & x.x \\
\hline
\end{tabular}

Table 2: Number of students taking / passing $C$ subjects during the two first academic semesters.

\begin{tabular}{|l|c|c|c|c|}
\hline$C$ & 1st Sem. & Pass & 2nd Sem. & Pass \\
\hline 0 & - & 6069 & - & 3924 \\
\hline 1 & 814 & 3155 & 1289 & 2641 \\
\hline 2 & 5518 & 5190 & 5760 & 4284 \\
\hline 3 & 9451 & 5102 & 5286 & 3367 \\
\hline 4 & 4181 & 1692 & 2557 & 1450 \\
\hline 5 & 1130 & 388 & 929 & 463 \\
\hline 6 & 521 & 150 & 354 & 167 \\
\hline 7 or more & 177 & 46 & 181 & 60 \\
\hline Total & 21792 & 15723 & 16356 & 12432 \\
\hline \hline Mean & 3.073 & 1.78 & 2.873 & 1.888 \\
\hline Median & 3 & 2 & 3 & 2 \\
\hline 1st-3rd Qs. & {$[1,4]$} & {$[0,3]$} & {$[1,3]$} & {$[1,3]$} \\
\hline
\end{tabular}

coming a good "e-learner" depends not only on the student herself but also on the institutional support, so the more information the learners have, the better decisions they might take.

On the other hand, Table 2 shows also that, in the first semester, most students fail to pass all subjects they enrolled into. Preliminary results show that the most important variable for predicting dropping out after the first semester is the number of subjects successfully taken, so adjusting the number of subjects taken during the first semester becomes a key issue, for both the institution and the students. One of the main differences between brick-and-mortar universities and distance ones is that the latter have more flexible requirements: no minimum enrollment, possibility of taking one or more consecutive semester breaks, and so. But, are students following the institutional recommendations? We propose to analyze the subjects taken by students, in order to determine the most common subject combinations and try to visually detect any relationship with the fact of passing or not a subject. We call this "intra-semester analysis". We are also interested in analyzing how students advance within a given programme, that is, which subjects they will enroll in the next semester according to the results of the preceding one. We call this "intersemester analysis". 
Table 3: Number of students $(N)$ taking / passing a given subject the first academic semester sorted by popularity.

\begin{tabular}{|l|l|c|c|c|c|}
\hline Rank & subject & $N$ & Pass & Acum. & Pct. \\
\hline 1 & 00.010 & 15229 & 10500 & 15229 & $69.9 \%$ \\
\hline 2 & 01.001 & 7433 & 4519 & 17566 & $80.6 \%$ \\
\hline 3 & 01.079 & 6662 & 3481 & 18528 & $85.0 \%$ \\
\hline 4 & $01.005^{*}$ & 6615 & 3698 & 19328 & $88.7 \%$ \\
\hline 5 & 00.002 & 4654 & 2621 & 19795 & $90.8 \%$ \\
\hline$\ldots$ & $\ldots$ & $\ldots$ & $\ldots$ & $\ldots$ & $\ldots$ \\
\hline 10 & $01.009^{*}$ & 1432 & 756 & 20790 & $95.4 \%$ \\
\hline$\ldots$ & $\ldots$ & $\ldots$ & $\ldots$ & $\ldots$ & $\ldots$ \\
\hline 18 & $01.020^{*}$ & 527 & 233 & 21562 & $98.9 \%$ \\
\hline
\end{tabular}

\subsection{Intra-semester analysis}

Table 3 shows the most popular subjects taken by students in their first academic semester. From the total pool of 501 possible subjects, students choose only among 128, following a long-tail distribution. Roughly, $90 \%$ percent of the students take at least one of the first five most popular subjects, $95 \%$ of the students take one of the first ten most popular subjects and $99 \%$ take one of the first eighteen. These will be the subjects selected for the visualization using chord diagrams, in order to see whether the density of the graph is appropriate enough to include as much as information as possible without being too complex. Notice also that there are students enrolling into subjects that are not supposed to be taken during the first academic semester (those marked in Table 3 with *). This is a typical situation in distance universities where students are not compelled to enroll a predetermined set of subjects.

Table 4 reproduces the same analysis but for the most popular subjects taken in the second academic semester. Notice that in this case, "second" has a more complex definition, as we take into account breaks taken by students. For instance, if a student takes a break during her second semester, the next semester she is taking a subject is used as her second one. Notice also that, as some students drop out after the first semester, the number of students is smaller (16356). Compared to Table 3, the accumulated percentage of students taking one of the most popular subjects grows more slowly. In fact, students choose among 181 subjects out of the total pool of 501, so the number of possible combinations is larger than in the first academic semester. Therefore, in order to analyze the same percentage of students $(90 \%, 95 \%$ and $99 \%$ ) it is necessary to include more subjects in the visualization (12, 16 and 24 respectively).

This analysis can be repeated for the third and further semesters. In summary, in the third semester, at least one of the 15,18 and 26 most popular subjects is taken by the $90 \%, 95 \%$ and $99 \%$ of the total students
Table 4: Number of students $(N)$ taking / passing a given subject the second academic semester sorted by popularity.

\begin{tabular}{|l|l|c|c|c|c|}
\hline Rank & subject & $N$ & Pass & Acum. & Pct. \\
\hline 1 & 01.005 & 4022 & 2470 & 4022 & $24.6 \%$ \\
\hline 2 & 01.006 & 3704 & 2699 & 6611 & $40.4 \%$ \\
\hline 3 & $01.079^{*}$ & 3369 & 2005 & 8670 & $53.0 \%$ \\
\hline 4 & $01.001^{*}$ & 3362 & 2135 & 10266 & $62.8 \%$ \\
\hline 5 & 01.078 & 2942 & 2247 & 11172 & $68.3 \%$ \\
\hline$\ldots$ & $\ldots$ & $\ldots$ & $\ldots$ & $\ldots$ & $\ldots$ \\
\hline 12 & $00.002^{*}$ & 1827 & 1087 & 14810 & $90.5 \%$ \\
\hline$\ldots$ & $\ldots$ & $\ldots$ & $\ldots$ & $\ldots$ & $\ldots$ \\
\hline 16 & $00.004^{*}$ & 1299 & 1059 & 15665 & $95.8 \%$ \\
\hline$\ldots$ & $\ldots$ & $\ldots$ & $\ldots$ & $\ldots$ & $\ldots$ \\
\hline 24 & $01.012^{*}$ & 481 & 333 & 16185 & $99.0 \%$ \\
\hline
\end{tabular}

Table 5: Most popular combinations of subjects taken in consecutive enrollments.

\begin{tabular}{|c|c|c|c|c|c|}
\hline subject & 01.005 & 01.006 & 01.079 & 01.001 & 01.078 \\
\hline 00.010 & 3230 & 3026 & 2533 & 2654 & 2061 \\
\hline 01.001 & 1915 & 1711 & 1473 & 898 & 1447 \\
\hline 01.079 & 1597 & 1434 & 1048 & 1166 & 1775 \\
\hline 01.005 & 1075 & 1398 & 1178 & 1321 & 886 \\
\hline 00.002 & 915 & 807 & 725 & 767 & 644 \\
\hline
\end{tabular}

(13718) from a pool of 200 subjects. In the fourth semester, figures are 16, 20 and 30 respectively, for a total of 11831 students and 191 different subjects. Notice that 30 different subjects generate $\left(\begin{array}{c}30 \\ 2\end{array}\right)$ different combinations (i.e. 435), which is probably a figure too large for visualization purposes. Therefore, in the following sections we will use the $95 \%$ level for visualizing relationships between subjects.

\subsection{Inter-semester analysis}

In this case we are interested in analyzing the sequence of subjects taken by students, that is, what subjects they enroll into once they know the results of the previous semester. Usually, when a student fails to pass a subject, she is more likely to enroll into such subject again the next semester, altogether with other new subjects, following (or, unfortunately, not) the recommendations given by the university (Table 1).

Table 5 shows, for each subject in the first semester (rows), the number of students that take a specific subject the second semester (columns), for the first five most popular subjects each semester. Only $65.3 \%$ of the 16356 students take one of these 25 combinations. In order to represent the $95 \%$ of students' enrollments, this table should have at least, 10 rows $\times 16$ columns, which is clearly unwieldy. 


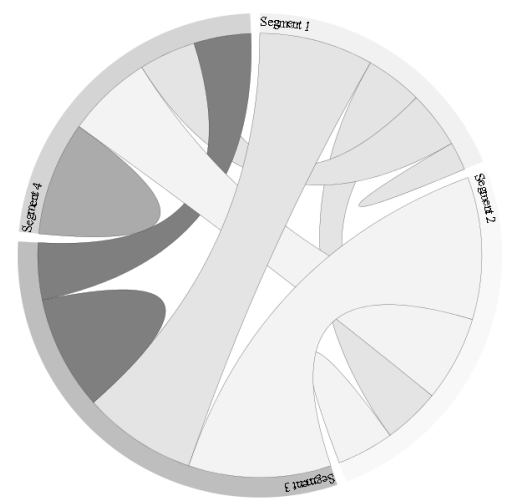

Figure 1: Example of chord diagram.

\section{VISUALIZING ENROLLMENT DATA}

Visualizing large volumes of data is not a trivial problem, specially when several dimensions are involved (temporal, relationships among elements, and so). As stated by (Shneiderman, 1996), it is very important to find the appropriate visual representations of different types of relationships between data entries. Among the huge amount of possibilities (see (McCandless, 2009; Yau, 2011)), we have chosen to explore radial visualizations (Draper et al., 2009) as they are well suited for describing relationships between hierarchical data. We will focus in visualizing adjacency edges (i.e. relationships between subjects), following the approach described in (Holten, 2006), as well as considering the e-learning visualization context (Gómez-Aguilar et al., 2010).

\subsection{Chord diagrams}

In the light of the results described in the previous section, we propose to use chord diagrams for visualizing both intra-semester and inter-semester data (not included due to paper length restrictions). A chord diagram is composed by segments, namely nodes and chords. Nodes are arranged radially, drawing thick curves (i.e. chords) between them. The thickness of the curve encodes the frequency of a given aspect between the two connected nodes. In our case, each node is a subject, the more students take a subject, the bigger the node is, while chords between nodes represent the number of students taking both subjects at the same time.

For building chord diagrams we used D3.js, a Javascript library for manipulating data (Bostock et al., 2011). The aspect of a chord diagram is determined by the following variables: the number of subjects $N$, a vector of $N$ elements containing sev-

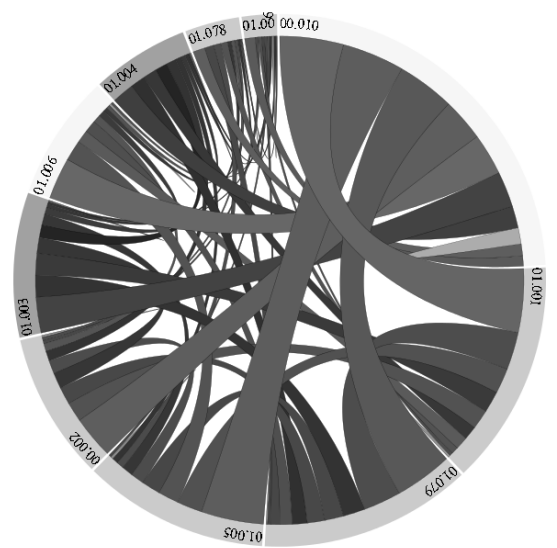

Figure 2: Relationships between the 10 most popular subjects in the first academic semester.

eral attributes for each subject (in our case, number of students and success rate, which will determine node size and color respectively), a $N \times N$ adjacency matrix containing elements in the form of $N^{i j}=N_{00}^{i j}+N_{01}^{i j}+N_{10}^{i j}+N_{11}^{i j}$ where $N^{i j}$ is the number of students simultaneously taking subjects $i$ and $j$ and subscripts describe the $2 \times 2$ matrix containing the number of students failing/passing (0/1) each subject respectively. Due to printing restrictions, chord diagrams are shown here using gray tones only. Interactive chord diagrams use color for increasing the ability of discovering patterns: success rate $[0,1]$ is mapped to a continuous [red, green] interval (containing yellow). Such interval could be quantized into three bins $[0, a),[a, b)$ and $[b, 1](a<b)$ representing "under average", "average" and "above average", respectively. More complex coloring strategies could be designed as well, specially when visualizing subject combinations as chords.

\subsection{Visualization of intra-semester data}

Figure 2 shows the generated chord diagram for the data described in Table 3, for the 10 most popular subjects of the first semester. We have used $a=0.4$ and $b=0.6$ for quantizing node colors. subjects are in clockwise order according to the number of students taking each subject. On the other hand, Figure 3 reproduces the same visualization for the 16 most popular subjects of the second semester. In this case we show only the chords starting from a particular subject, as an example of the interaction described in Section 3.4. The complexity of these diagrams with 10 and 16 subjects is quite high, so $90 \%$ will be probably a better level for exploration purposes, including more subjects dynamically if desired. 


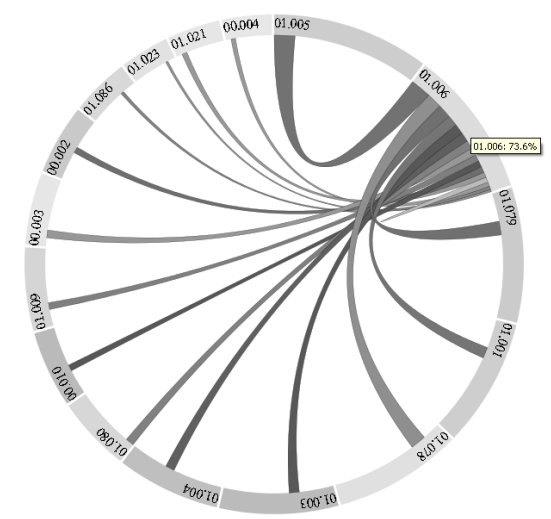

Figure 3: Relationships between the 16 most popular subjects in the second academic semester, showing some data only for a given subject.

\subsection{Visualization of inter-semester data}

In this case we will think of chord diagrams as having two halves: the left one contains the subjects from the first semester, while the right one contains the subjects from the second one. Obviously some subjects can be repeated in both halves (i.e. students taking the same subject again), but no chords will be drawn between subjects on the same half. Actually, it is like visualizing a bipartite graph but maintaining the same metaphor. Segment width and color follow the same rules described in the previous sections. Chord width is determined by the number of students taking one subject from the right half after having taken one from the left one. Chord color can be determined by the percentage of students successfully passing both subjects, showing dangerous/suitable subject enrollment sequences. Figure 4 shows the chord diagram generated with the data in Table 5. This diagram could also be created trying to reproduce the institutional recommendations (Table 1), including one more subject every semester, in order to see reality compared to the predetermined programme sequence. Nevertheless, as some of the most popular subjects in the second semester are from the first one (because students have not taken them yet or they have but failed to do so), the number of subjects should be larger in the right half of the chord diagram, increasing its complexity.

\subsection{Adding interaction}

Following Shneiderman's mantra, "overview first, zoom and filter, then details on demand" (Shneiderman, 1996), we can use the possibilities of D3.js to add some interaction to chord diagrams. While the basic chord diagram (as shown in Figure 2) would be the first task ("overview"), it is possible to move

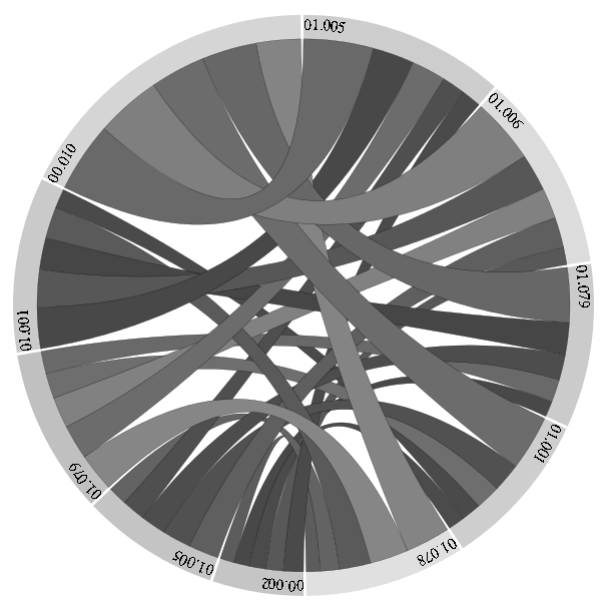

Figure 4: Relationships between the 5 most popular subjects each semester seen as consecutive enrollments.

forward the second task ("zoom and filter") and the third one ("details on demand") using the following approach:

- "zoom and filter": if the user moves the cursor (i.e. the mouse) over any segment, only those other segments directly connected to such segment are visualized, the rest are filtered out by fading. Therefore, if the user moves the cursor over a node, only the node and the chords connecting such node to other nodes are visible. On the other hand, if the user moves the cursor over a chord, only that chord and the two nodes connected by such chord are visible. Zoom can help users to perform the "filter" task for small segments.

- "details on demand": if the user selects (i.e. by clicking) any segment, a small window containing information about such segment is displayed. If the segment is a node, data about such subject is displayed, namely its code, name, academic semester, percentage of students passing it and percentage of students taking it for second and further times. If the segment is a chord, the percentage of students taking the two subjects simultaneously (or consecutively) as well as the $2 \times 2$ matrix with the pass/fail rates are shown.

\section{CONCLUSIONS}

Enrollment in educational institutions with flexible requirements (such as distance and online universities) does not follow uniform patterns with respect to the subjects each student enrolls into or with respect to the recommended sequence proposed by the 
institution. The concept of cohort (students taking the same subjects and advancing within a programme at the same pace) is completely unsuitable. Students get scattered between semesters, so their only nexus are subjects taken simultaneously. Therefore, programme planning becomes a complex issue involving large tables of numbers which are too large to comprehend by university managers. On the other hand, students have no support for determining which combinations of subjects are more suitable, specially in their first academic semester when they have no experience in what means being an online learner.

In this paper we have described the use of chord diagrams for visualizing intra-semester enrollment data, namely the combinations of subjects taken by students simultaneously. The number of subjects (and their relationships) visualized as chord diagrams is determined by a threshold, trying to capture as many students as possible. We have created diagrams that include $95 \%$ of the students, but they are quite complex, so probably a threshold of $90 \%$ is enough for exploration purposes. Currently now, university managers are using tabular data for detecting programme bottlenecks; we expect to introduce and evaluate the use of chord diagrams as a simple way to visualize such information as part of an internal institutional innovation project. These visualizations will be part of an enrollment support system that will guide students and their mentors through the large amount of subject combinations, according to both their personal interests and background, but taking into account the academic performance of previous students with similar enrollment patterns.

Current and future work in this topic should include the creation of more complex visualizations involving more than two semesters, using concentric chords or a 3D version, arranging chords in an imaginary 3D cone or cylinder. More experimentation for determining the best number of subjects and the coloring scheme is also needed, as well as the information provided by the interaction with the chord diagram.

\section{Acknowledgments}

This work is supported by Spanish Ministry of Science and Innovation project MAVSEL (ref. TIN2010-21715-C02-02).

\section{REFERENCES}

Bostock, M., Ogievetsky, V., and Heer, J. (2011). D $\mathrm{D}^{3}$ datadriven documents. IEEE Transactions on Visualiza- tion and Computer Graphics, 17(12):2301-2309.

Campbell, J., DeBlois, P., and Oblinger, D. (2007). Academic analytics: A new tool for a new era of educational research. EUCAUSE Review, 42(4):40-57.

Card, S. K., Mackinlay, J. D., and Shneiderman, B. (1999). Readings in information visualization - using vision to think. Academic Press.

Draper, G. M., Livnat, Y., and Riesenfeld, R. F. (2009). A survey of radial methods for information visualization. IEEE Transactions on Visualization and Computer Graphics, 15(5):759-776.

Gómez-Aguilar, D. A., Suárez-Guerrero, C., TheronSánchez, R., and García-Peñalvo, F. (2010). Visual analytics to support e-learning. In Advances in Learning Processes.

Grau-Valldosera, J. and Minguillón, J. (2014). Rethinking dropout in online higher education: The case of the universitat oberta de catalunya. The International Review of Research in Open and Distance Learning, $15(1)$.

Holten, D. (2006). Hierarchical edge bundles: Visualization of adjacency relations in hierarchical data. IEEE Transactions on Visualization and Computer Graphics, 12(5):741-748.

Kiernan, M., Woodroffe, M., and Thomas, P. (2004). Open 24/7: The journey from e-user to e-learner. In Nall, J. and Robson, R., editors, Proceedings of World Conference on E-Learning in Corporate, Government, Healthcare, and Higher Education 2004, pages 9597, Washington, DC, USA. AACE.

McCandless, D. (2009). Information is beautiful. Collins, London.

Park, J.-H. and Choi, H. J. (2009). Factors influencing adult learners' decision to drop out or persist in online learning. Educational Technology \& Society, 12(4):207217

Sacín, C. V., Chue, J., Peche, J. P., Alvarado, G., Vinatea, B., Estrella, J., and Ortigosa, A. (2011). A data mining approach to guide students through the enrollment process based on academic performance. User Modeling and User-Adapted Interaction, 21(1-2):217-248.

Shneiderman, B. (1996). The eyes have it: A task by data type taxonomy for information visualizations. In Proceedings of the IEEE Symposium on Visual Languages, pages 336-343.

Siraj, F. and Abdoulha, M. A. (2009). Uncovering hidden information within university's student enrollment data using data mining. In Al-Dabass, D., Triweko, R., Susanto, S., and Abraham, A., editors, Asia International Conference on Modelling and Simulation, pages 413-418. IEEE Computer Society.

Tyler-Smith, K. (2006). Early attrition among first time elearners: A review of factors that contribute to dropout, withdrawal and non-completion rates of adult learners undertaking elearning programmes. Journal of Online Learning and Teaching, 2(2).

Yau, N. (2011). Visualize This: The FlowingData Guide to Design, Visualization, and Statistics. John Wiley \& Sons. 